\title{
State Estimation of Drive-by-wire Chassis Vehicle Based on Dual Unscented Particle Filter Algorithm
}

\section{Zixu Wang}

Jilin University State Key Laboratory of Automotive Simulation and Control

\section{Chaoning Chen}

Jilin University State Key Laboratory of Automotive Simulation and Control

\section{Quan Jiang}

Jilin University State Key Laboratory of Automotive Simulation and Control

Hongyu Zheng ( $\nabla$ zhenghy@jlu.edu.cn )

Jilin University State Key Laboratory of Automotive Simulation and Control https://orcid.org/00000002-4405-2094

\section{Chuyo Kaku}

Jiang su Chaoli Electric CO Ltd

\section{Research Article}

Keywords: Drive-by-wire chassis vehicle, Vehicle state estimation, Dual unscented particle filter, Tire force estimation, Unscented particle filter

Posted Date: February 15th, 2022

DOI: https://doi.org/10.21203/rs.3.rs-1279402/v1

License: (c) (i) This work is licensed under a Creative Commons Attribution 4.0 International License.

Read Full License 


\section{Title page}

\section{State estimation of drive-by-wire chassis vehicle based on dual unscented particle filter algorithm}

Zi-Xu Wang, born in 1999, is currently a master candidate at State Key Laboratory of Automotive Simulation and Control, Jilin University, Changchun, China

Tel: +86-18343526155; E-mail: 345197599@qq.com

Chao-Ning Chen, born in 1999, is currently a Ph.D candidate at State Key Laboratory of Automotive Simulation and Control, Jilin University, Changchun, China.

E-mail:1176660329@qq.com

Quan Jiang, born in 1997, is currently a master candidate at State Key Laboratory of Automotive Simulation and Control, Jilin University, Changchun, China

E-mail:1049819809@qq.com@163.com

Hong-Yu Zheng, born in 1980, received the B.E. degree in mechanical engineering and automation and the Ph.D.degree in vehicle engineering from Jilin University, Changchun, China, in 2003 and 2009, respectively. From October 2017 to October 2018, he was a Visiting Research Scholar with the Department of Mechanical and Aerospace Engineering, The Ohio State University, Columbus, OH, USA. He is currently a Professor with the State Key Laboratory of Automotive Simulation and Control, Jilin University. His research interests include vehicle dynamics and control and control of autonomous vehicles.

E-mail: zhenghy@jlu.edu.cn

Chuyo Kaku, born in 1962, received the Ph.D.degree in mechanical engineering from Tokyo Institute of Technology, Tokyo, Japan, in 1995 and 1998.

E-mail: guo.kaku@ chaoli-electric.com

\section{Corresponding author: Hong-Yu Zheng E-mail: zhenghy@jlu.edu.cn}




\title{
State estimation of drive-by-wire chassis vehicle based on dual unscented particle filter algorithm
}

\author{
Zi-Xu Wang ${ }^{1}$, Chao-Ning Chen ${ }^{1}$, Quan Jiang ${ }^{1}$, Hong-Yu Zheng ${ }^{1}$, CHUYO KAKU ${ }^{2}$
}

Received June xx, 201x; revised February xx, 201x; accepted March xx, 201x

(c) Chinese Mechanical Engineering Society and Springer-Verlag Berlin Heidelberg 2017

\begin{abstract}
Vehicle dynamic state parameter information plays an important role in vehicle driving safety, and accurate vehicle dynamic information can improve the dynamic performance of the controlled vehicle. This paper proposes a state estimation method for drive-by-wire chassis vehicle based on the dual unscented particle filter algorithm, which make full use of the known advantages of the four-wheel drive torque and steer angle parameters of the drive-by-wire chassis vehicle. In the dual unscented particle filter algorithm, two unscented particle filter transfer information to each other, observe the vehicle state information and the tire force parameter information of the four wheels respectively, which reduce the influence of parameter uncertainty and model parameter changes on the estimation accuracy during driving. Dual unscented particle filter algorithm is verified through both simulation and experiment. The performance with the proposed algorithm is analyzed in terms of the time-average square error in double lane change as compared to the case where unscented Kalman filter is utilized. The results demonstrate the effectiveness of the proposed algorithm.
\end{abstract}

Keywords: Drive-by-wire chassis vehicle - Vehicle state estimation - Dual unscented particle filter - Tire force estimation - Unscented particle filter

\section{Introduction}

With rapid development of wire control technology has

Hong-Yu Zheng

zhenghy@jlu.edu.cn

1 State Key Laboratory of Automotive Simulation and Control, Jilin University, Changchun 130022, China

2 Jiangsu Chaoli Electric Co. Ltd, Danyang, Jiangsu, 212321, China made the drive-by-wire chassis vehicle with the advantages of precise knowledge of the four-wheel drive torque and independent control of the four-wheel steer angle have become the research focus [1]. At the same time, various vehicle active safety control systems, such as yaw stability control, lane departure avoidance system, and collision avoidance system, direct yaw moment control etc. [2-5], require accurate vehicle state information, which makes it important to accurately obtain vehicle driving state information for the safety of vehicles. The high precision wheel drive torque and the wheel steer angle information of the drive-by-wire chassis vehicle is of great significance to the estimation of the vehicle driving state.

At present, the research on state estimation of traditional vehicles is mainly based on Kalman algorithms and its derivative algorithms. Zong et al. [6-7] used the extended Kalman filter (EKF) algorithm to measure the vehicle state to obtain the vehicle speed, and conducts offline simulation based on the actual vehicle measurement data. Zhao et al. [8] proposed the unscented Kalman filter (UKF) algorithms to observe the vehicle speed under typical operating conditions in real time. The test results show that the algorithm has high accuracy and robustness. Cui et al. [9] presented the UKF algorithm to estimate lateral tire force, tire slip angle, vehicle sideslip angle and road friction coefficient, UKF estimator is accurate in different road conditions. Aiming at the vehicle lateral velocity estimation method (VLVEM), Zhang et al. [10] derived a Stack Bidirectional Long Short-Term Memory (SBI-LSTM) algorithm based on the federated Kalman filter (FKF) algorithm proved by simulations that has the characteristics of accurate estimation, robustness and fault tolerance. Zhang et al. [11] proposed adaptive treble extend Kalman filter and designed a sliding mode update, which make the 
overall system becomes more robust for tire force estimation. The results show that the algorithm achieves an excellent performance in a vertical force evaluation.

On the other hand, because the particle filter algorithm does not require the assumption of Gaussian distribution and has the advantage of being able to deal with high-order nonlinear problems, it has begun to be applied to the research of vehicle state estimation. Lenzo et al. [12] investigated a particle filter (PF) approach to estimate the chassis sideslip angle of road vehicle, the results show that the performance of the algorithm is satisfactory. However, the PF algorithm also has the disadvantages of slow convergence speed and large amount of calculation [13]. Ko et al. [14] compared the performance of PF and EKF in the position estimation of underwater robots and pointed out that the result of PF is smoother and more stable, but the calculation speed is slower. Furthermore, the general PF also has: (1) Particle degradation: After multiple sampling, the particles will be concentrated near the higher probability density value, causing the update invalid; (2) Sample size dependence: There is a strong conflict between particle filter calculation efficiency and calculation accuracy. If the number of particles is insufficient, the estimation result would be distorted [15]. Berntorp [16] compared the time average root mean square error probability of different particle numbers in the research and illustrated that the number of particles is inversely proportional to the measurement error.

In order to solve the above problems, some researchers combine Kalman and its derivative filtering theory with PF. For example, the unscented particle filter (UPF) algorithm is based on the particle filter framework and uses the algorithm of UKF to generate the importance function, and incorporates the latest observational information in the prior update. Liu et al. [17] proposed a UPF with fine resampling to estimate tire slip angle and yaw rate, the simulation proved that its robustness is higher than UKF, and the fluctuation of the error value is smaller than sequential importance resampling particle filter (SIR-PF). Aiming at the problem of improving the accuracy of vehicle positioning, Lin et al. [18] used a particle-assisted unscented Kalman filter (PAUKF), in which UKF algorithm updates the vehicle state and PF algorithm provides additional positioning information. Liu et al. [19] presented a road friction coefficient estimation method that combines the auxiliary particle filter and the iterative extended Kalman filter (APF-IEKF), and used the iterative algorithm to process the results of the first step, and the simulation and experiment in low tire road coefficient (snow course) in winter were provided to demonstrate the accuracy and efficiency of the proposed approach.

The current research on the state estimation problem of the drive-by-wire chassis vehicle is based on Kalman and PF and the derivative algorithm. Song et al. [20] built a chassis controller for the direct yaw moment control (DYC) system of a four-wheel independent steer (4WIS) vehicle based on the UKF estimation of the vehicle tire slip angle, longitudinal speed and tire force. Chu et al. [21] applied the UPF algorithm to estimate the lateral tire force, longitudinal velocity, lateral velocity and yaw rate of the in-wheel-motor vehicle, verifying its effectiveness and robustness. Zhu et al. [22] proposed an improved particle filter to estimate in-wheel-motor vehicle state parameters under complex noise and sensor failure conditions, and the simulation results show that its performance is better than UPF and UKF observers.

Since the drive-by-wire chassis vehicle has the advantages of drive torque and steer angle parameters accurate controllability, it is considered to use two observers to process different state vectors, and to observe the vehicle state parameters in cooperation with each other. Such an algorithm structure reduces parameter uncertainty and interference caused by model parameter changes and improve the accuracy of the observed measurement. Pei et al. [23] presented a joint estimation method of vehicle state and parameters based on dual unscented Kalman filter (DUKF), which has a high estimation accuracy for the vehicle state and parameters of $8 \times 8$ distributed electric vehicle. Wang et al. [24] designed a combination of UKF and EKF algorithm for the estimation of tire sideslip angles of four-wheel independent drive (4WID) electric vehicle and a hybrid estimator was formed through weighting factors with strong robustness.

In this paper, a dual unscented particle filter (DUPF) algorithm is proposed to estimate the driving state parameters of the drive-by-wire chassis vehicle. The main contributions of this article are:

1. For the estimation of the driving state of the drive-by-wire chassis vehicle, the DUPF algorithm is applied which is based on the DUKF algorithm framework and PF particles. Two UPF observer state vectors are respectively selected as vehicle state information and vehicle parameter information, which reduces parameter uncertainty and the influence of model parameter changes during driving on the estimation results, and improves the estimation accuracy of vehicle state parameters and the robustness of the algorithm.

2. According to the characteristics of easy acquisition of wheel angle parameters and torque parameters of the drive-by-wire chassis vehicle, combined with low-cost 
sensors, an accurate estimation of the vehicle state can be performed. The algorithm is verified through both double lane change (DLC) and Weave test.

The rest of this article is structured as follows: In the second section introduces a nonlinear drive-by-wire chassis vehicle model and Pacejka tire model. In the third section introduces the DUPF algorithm that used for vehicle state and parameter estimation. In the fourth section introduces the simulation and driving simulator test under the DLC and weave test. In the fifth section is the conclusion of the article.

\section{Models and estimation algorithm}

\subsection{Drive-by-wire chassis vehicle dynamic model}

As shown in Figure 1, $\alpha_{i j}$ is wheel slip angle, $\delta_{i j}$ is the wheel steer angle $(i$ can be $l, r$ represent left and right; $j$ can be $f, r$ represent front and rear), $V_{x}, V_{y}$ is the longitudinal speed and lateral speed of the center of mass of the vehicle in the body coordinate system, $a_{x}, a_{y}$ is the longitudinal acceleration and lateral acceleration of the center of mass of the vehicle in the body coordinate system, $\beta$ is the slip angle of the center of mass, $\theta$ is the wheel forward direction angle.

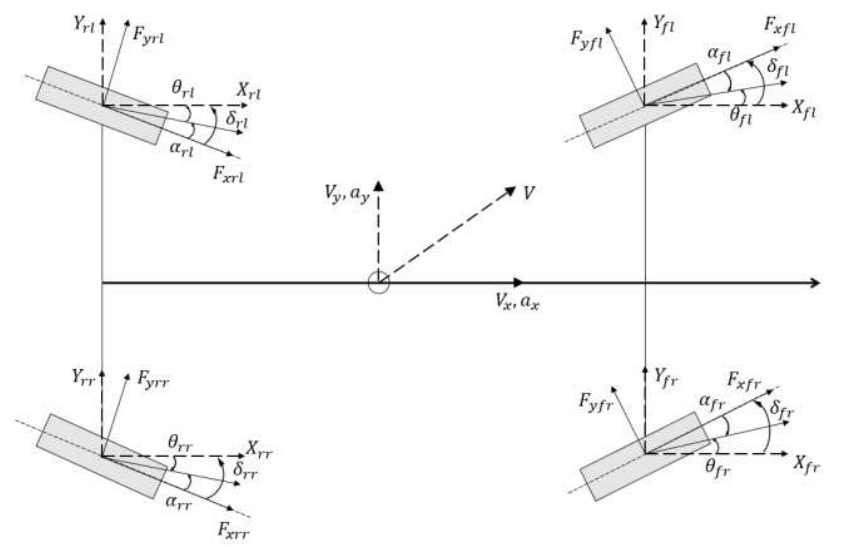

Figure 1 Drive-by-wire chassis vehicle dynamic model Wheel slip angle can be expressed as:

$$
\begin{aligned}
& =-\delta_{i j} \\
& +\theta_{i j}
\end{aligned}
$$

The vehicle state parameter estimator proposed in this paper is based on a nonlinear drive-by-wire chassis vehicle dynamics model, which contains three degrees of freedom in the longitudinal, lateral and yaw directions. $F_{x i j}, F_{y i j}$ represent the longitudinal and lateral tire force in the wheel coordinate system. $X_{i j}, Y_{i j}$ are the longitudinal component force and the lateral component force received at the center of the wheel in the body coordinate system as:

$$
\left[\begin{array}{c}
X_{i j} \\
Y_{i j}
\end{array}\right]=R\left(\delta_{i j}\right)\left[\begin{array}{l}
F_{x_{-} i j} \\
F_{y_{-} i j}
\end{array}\right]=\left[\begin{array}{cc}
\cos \delta_{i j} & -\sin \delta_{i j} \\
\sin \delta_{i j} & \cos \delta_{i j}
\end{array}\right]\left[\begin{array}{c}
F_{x_{i j}} \\
F_{y_{i j}}
\end{array}\right]
$$

In addition to the tire force, the air resistance experienced by the vehicle during driving is:

$F_{d}=\frac{1}{2} \rho C_{d} A v_{x}^{2}$

Where $\rho$ is air density, $C_{d}$ is drag coefficient, $A$ is frontal area. Therefore, the motion equations of the vehicle in the longitudinal, lateral and yaw directions is:

$$
\left\{\begin{array}{c}
m a_{x}=m\left(\dot{V}_{x}-\dot{\varphi} V_{y}\right)=\sum_{i=l}^{r} \sum_{j=f}^{r} X_{i j}-F_{d} \\
m a_{y}=m\left(\dot{V}_{y}-\dot{\varphi} V_{x}\right)=\sum_{i=l}^{r} \sum_{j=f}^{r} Y_{i j} \\
I_{z} \ddot{\varphi}=\frac{t}{2}\left(-X_{l f}+X_{r f}-X_{l r}+X_{r r}\right) \\
+l_{f}\left(Y_{l f}+Y_{r f}\right)-l_{r}\left(Y_{l r}+Y_{r r}\right)
\end{array}\right.
$$

Where $\dot{\varphi}$ is yaw rate, $l_{f}, l_{r}$ are the distance from the center of mass to the front axle and rear axle. In the case of considering wheel load transfer, the vertical load of each wheel is:

$$
\left\{\begin{array}{l}
F_{z l f}=\frac{m g l_{r}}{2\left(l_{f}+l_{r}\right)}-\frac{m_{s} a_{x} h_{s}}{2\left(l_{f}+l_{r}\right)}-\frac{m_{s} a_{y} h_{s}}{t_{f}} \\
F_{z r f}=\frac{m g l_{r}}{2\left(l_{f}+l_{r}\right)}-\frac{m_{s} a_{x} h_{s}}{2\left(l_{f}+l_{r}\right)}+\frac{m_{s} a_{y} h_{s}}{t_{f}} \\
F_{z l r}=\frac{m g l_{f}}{2\left(l_{f}+l_{r}\right)}+\frac{m_{s} a_{x} h_{s}}{2\left(l_{f}+l_{r}\right)}-\frac{m_{s} a_{y} h_{s}}{t_{r}} \\
F_{z r r}=\frac{m g l_{f}}{2\left(l_{f}+l_{r}\right)}+\frac{m_{s} a_{x} h_{s}}{2\left(l_{f}+l_{r}\right)}+\frac{m_{s} a_{y} h_{s}}{t_{r}}
\end{array}\right.
$$

Where $m_{s}$ is sprung mass, $h_{s}$ is the height of the center of mass of the sprung mass. The sideslip angle $\beta$ can be calculated by longitudinal velocity and lateral velocity:

$=\arctan \left(\frac{V_{y}}{V_{x}}\right)$ 


\subsection{Pacejka tire model}

As an important part of the direct contact between the vehicle and the ground, the functional relationship between the tire force and the tire motion parameters is the basis of vehicle dynamics research. The commonly used tire models are mainly divided into three categories: physical models, empirical and semi-empirical models, and finite element models. Each tire model has its own advantages and disadvantages. In this paper, the Pacejka tire model is selected, and the model calculation formula is [25]:

$$
y=D \sin \{\operatorname{Carctan}[B x-E(B x-\arctan B X)]\}
$$

Where $y$ is lateral tire force, $x$ is tire slip angle, $B$ is stiffness coefficient, $C$ is shape coefficient, $D$ is the maximum point, $E$ is the curvature coefficient. The forward direction angle of each wheel can be obtained through tire kinematics:

$$
=\left[\begin{array}{r}
\arctan \left(\frac{V_{y}+\dot{\varphi} l_{f}}{V_{x}-\dot{\varphi} t / 2}\right) \\
\arctan \left(\frac{V_{y}+\dot{\varphi} l_{f}}{V_{x}+\dot{\varphi} t / 2}\right) \\
\arctan \left(\frac{V_{y}-\dot{\varphi} l_{f}}{V_{x}-\dot{\varphi} t / 2}\right) \\
\arctan \left(\frac{V_{y}+\dot{\varphi} l_{f}}{V_{x}+\dot{\varphi} t / 2}\right)
\end{array}\right]
$$

Then the vertical load of the tire can be solved according to equation (5), the tire slip angle can be obtained from equations (1) and (8), then the tire cornering force can be obtained.

\section{Dual unscented particle filter algorithm}

Two UPF observers operate separately and simultaneously in the DUPF algorithm. In this paper, the vehicle state estimator and the vehicle parameter estimator exchange and correct information with each other. Both UPF observers are composed of particle sampling, UT transformation, prediction update, and calculate weight resampling. The principle of DUPF is shown in the Figure 2.

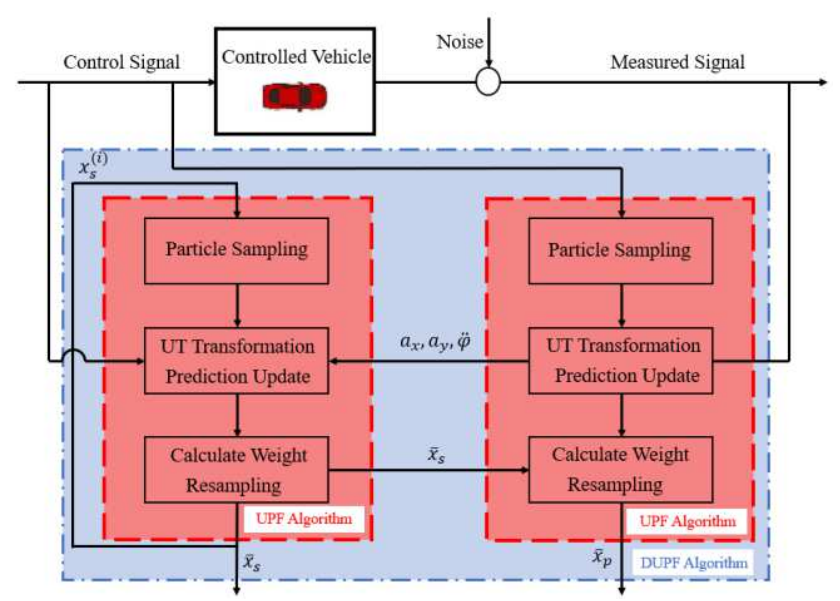

Figure 2 DUPF state observer algorithm logic diagram.

After discretizing the dynamic model of the vehicle and selecting the state vector and parameter vector, a vehicle state estimator based on the DUPF algorithm can be realized. The number of Sigma points depends on the number of state vectors and parameter vectors. In the DUPF algorithm, the vehicle dynamics model provides control variables, including each wheel torque, steer angle, wheel angular velocity and angular acceleration. The DUPF algorithm returns the observed vehicle yaw rate, vehicle longitudinal and lateral acceleration to the vehicle model.

\subsection{Unscented particle filter observer design}

In the research, only the movement of the vehicle on the horizontal plane is considered. According to the movement information of the vehicle in the longitudinal, lateral and yaw directions, the vehicle state is set to $x_{s}$, which is the observation vector of the DUPF observer:

$$
x_{s}=\left[\begin{array}{llllll}
V_{x} & V_{y} & \dot{\varphi} & a_{x} & a_{y} & \ddot{\varphi}
\end{array}\right]^{T}
$$

The vehicle parameter vector $x_{p}$ is:

$x_{p}=\left[\begin{array}{llllllll}F_{x l f} & F_{x r f} & F_{x l r} & F_{x r r} & F_{y l f} & F_{y r f} & F_{y l r} & F_{y r r}\end{array}\right]^{T}(10)$

Among them, $F_{x i j}, F_{y i j}$ respectively represent the longitudinal tire force and lateral tire force. Since the wheel steer angle and wheel torque signals of the drive-by-wire chassis vehicle can be directly obtained, the input vector of the observer: 
$u=\left[\begin{array}{llll}T_{w i j} & \delta_{i j} & \omega_{i j} & \dot{\omega}_{l \jmath}\end{array}\right]^{T}$

The observation vector is:

$y=\left[\begin{array}{lll}\dot{\varphi} & a_{x} & a_{y}\end{array}\right]$

Thus, there is the system discrete equation:

$$
\begin{gathered}
x_{s}(k+1)=f_{s}\left[x_{s}(k), x_{p}(k), u(k)\right]+Q_{s}(k) \\
y_{s}(k)=H_{s} x_{s}+v(k) \\
H_{s}=\left[\begin{array}{llllll}
0 & 0 & 1 & 0 & 0 & 0 \\
0 & 0 & 0 & 1 & 0 & 0 \\
0 & 0 & 0 & 0 & 1 & 0
\end{array}\right] \\
x_{p}(k+1)=f_{p}\left[x_{s}(k), u(k)\right]+Q_{p}(k) \\
y_{p}(k+1)=h_{p}\left[x_{p}(k+1), u(k+1)\right]+R(k+1)
\end{gathered}
$$

Where $\omega, Q_{s}, Q_{p}, v, R$ represents process noise and measurement noise respectively, and $Q_{s}, Q_{p}, R$ are diagonal matrix.

$$
\left\{\begin{array}{c}
V_{x}(k+1)=V_{x}(k)+\Delta T a_{x}(k) \\
V_{y}(k+1)=V_{y}(k)+\Delta T a_{y}(k) \\
\dot{\varphi}(k+1)=\dot{\varphi}(k)+\Delta T \ddot{\varphi}
\end{array}\right.
$$

Extract $N$ particles $\left\{x_{s 0}^{(i)}\right\}_{i=1}^{N},\left\{x_{p 0}^{(i)}\right\}_{i=1}^{N}$ from the prior distribution $p_{s}\left(x_{s 0}\right), p_{p}\left(x_{p 0}\right)$ respectively. Obtain the initial value of the state vector: $x_{s 0}^{(i)}, x_{p 0}^{(i)}$ and the initial covariance of the state vector: $P_{s 0}^{(i)}, P_{p 0}^{(i)}$, can be correspondingly expressed by:

$$
\begin{aligned}
& \bar{x}_{s}^{(i)}(0)=E\left[x_{s}^{(i)}(0)\right] \\
& P_{s}^{(i)}(0)=E\left[\left(x_{s}^{(i)}(0)-\bar{x}_{s}^{(i)}(0)\right)\left(x_{s}^{(i)}(0)-\bar{x}_{s}^{(i)}(0)\right)^{T}\right]
\end{aligned}
$$

$$
\begin{aligned}
& \left\{\begin{array}{l}
\chi_{s}^{(i) a}(k-1)=\left[\begin{array}{c}
\bar{x}_{s}^{(i) a}(k-1) \\
\bar{x}_{s}^{(i) a}(k-1) \pm \sqrt{\left(n_{a}+\lambda_{s}\right) P_{s}^{(i) a}(k-1)}
\end{array}\right]^{T} \\
\chi_{p}^{(i) a}(k-1)=\left[\begin{array}{c}
\bar{x}_{p}^{(i) a}(k-1) \\
\bar{x}_{p}^{(i) a}(k-1) \pm \sqrt{\left(n_{a}+\lambda_{p}\right) P_{p}^{(i) a}(k-1)}
\end{array}\right]^{T}
\end{array}\right. \\
& \lambda=\alpha^{2}(n+\kappa)-n \\
& \left\{\begin{array}{c}
W_{0}^{(m)}=\lambda /(L+\lambda) \\
W_{i}^{(m)}=\lambda / 2(L+\lambda)
\end{array}\right. \\
& \left\{\begin{array}{l}
W_{0}^{(c)}=\lambda /(L+\lambda)+\left(1-\alpha^{2}+\beta\right) \\
W_{i}^{(c)}=\lambda / 2(L+\lambda), i=1,2 \ldots, 2 L
\end{array}\right.
\end{aligned}
$$

Where $\kappa=0, \alpha=0.001, \beta=2$. After updating the Sigma point, $x_{s}^{(i) x}, x_{p}^{(i) x}$ as follow

$$
\begin{gathered}
x_{s}^{(i) x}(k \mid k-1)=f_{s}\left[x_{s}^{(i) x}(k-1), \hat{x}_{p}^{(i)}(k-1), u(k-1)\right] \\
x_{p}^{(i) x}(k \mid k-1)=f_{h}\left[\hat{x}_{s}^{(i)}(k-1), u(k-1)\right]
\end{gathered}
$$

Then the predicted value of the state vector $x_{s}, x_{p}$ obtained by weighted calculation is:

$$
\begin{aligned}
& \bar{x}_{s}^{(i)}(k \mid k-1)=\sum_{j=0}^{2 n_{s}} W_{s, j}^{(m)} x_{s, j}^{(i) x}(k \mid k-1) \\
& \bar{x}_{p}^{(i)}(k \mid k-1)=\sum_{j=0}^{2 n_{p}} W_{p, j}^{(m)} x_{p, j}^{(i) x}(k \mid k-1)
\end{aligned}
$$

Linear and non-linear changes are made to the Sigma point, there are system predicted values and covariance predicted values:

$$
\bar{y}_{s}^{(i)}(k \mid k-1)=\sum_{j=0}^{2 n_{s}} W_{s, j}^{(m)} y_{s}^{(i) x}(k \mid k-1)
$$

Calculate the Sigma point set: 


$$
\begin{aligned}
& P_{s(x, x)}^{(i)}(k \mid k-1) \\
& =\sum_{j=0}^{n_{s}} W_{s, j}^{(c)}\left[x_{s, j}^{(i)}(k \mid k-1)\right. \\
& \left.-\bar{x}_{s}^{(i)}(k \mid k-1)\right]\left[x_{s, j}^{(i)}(k \mid k-1)\right. \\
& -\bar{x}_{s}^{(i)}(k \mid k \\
& -1)]^{T}
\end{aligned}
$$

The updated filter gain matrix is:

$$
K_{S}(k)=P_{s(x, y)}(k)\left[P_{s(\tilde{y}, \tilde{y})}\right]^{-1}
$$

Then there are posterior estimates and posterior variance:

$$
\begin{gathered}
\bar{x}_{s}^{(i)}(k)=\bar{x}_{s}^{(i)}(k \mid k-1)+K_{s}(k)\left[y_{s}(k)-\bar{y}_{s}^{(i)}(k \mid k-1)\right] \\
\hat{P}_{s}^{(i)}(k)=P_{s(x, x)}^{(i)}(k \mid k-1)-K_{s}(k) P_{s(\tilde{y}, \tilde{y})}(k)\left[K_{s}(k)\right]^{T}
\end{gathered}
$$

Furthermore, $\hat{x}_{p}^{(i)}(k), \hat{P}_{p}^{(i)}(k)$ can be obtained by the same method.

For each particle:

$$
\begin{array}{r}
\hat{x}_{s}^{(i)}(k) \sim q\left(\hat{x}_{s}^{(i)}(k) \mid x_{s}^{(i)}(0: k-1), y_{s}(1: k)\right) \\
=N\left(\bar{x}_{s}^{(i)}(k), \hat{P}_{s}^{(i)}(k)\right) \\
\hat{x}_{s}^{(i)}(0: k) \triangleq\left(x_{s}^{(i)}(0: k-1), \hat{x}_{s}^{(i)}(k)\right)
\end{array}
$$

The weight of each particle is:

$$
\propto \frac{p\left(y_{s}(k) \mid \hat{x}_{s}^{(i)}(k)\right) p\left(\hat{x}_{s}^{(i)}(k) \mid x_{s}^{(i)}(k-1)\right)}{q\left(\hat{x}_{s}^{(i)}(k) \mid x_{s}^{(i)}(0: k-1), y_{s}(1: k)\right)}
$$

Particle normalization:

$$
\widetilde{\omega}_{s}^{(i)}(k)=\omega_{s}^{(i)}(k) / \sum_{j=1}^{N} \omega_{s}^{(j)}(k)
$$

\subsection{Resampling}

After multiple iterations, most of the particle weights in the PF algorithm will become 0 . It is necessary to replicate high-weight particles through resampling and eliminate low-weight particles. Recording the weights of each particle as:

$$
\omega_{s}^{(i)}(k)=1 / N
$$

Therefore, the output state vector is:

$$
\begin{aligned}
\hat{x}_{s}^{(i)}(k)= & \sum_{i=1}^{N} \omega_{s}^{(i)}(k) \bar{x}_{s}^{(i)}(k) \\
& \hat{P}_{s}(k)=\sum_{i=1}^{N} \omega_{s}^{(i)}(k)\left[x_{s}^{(i)}(k)\right. \\
& \left.-\bar{x}_{s}^{(i)}(k)\right]\left[x_{s}^{(i)}(k)\right. \\
& \left.-\bar{x}_{s}^{(i)}(k)\right]^{T}
\end{aligned}
$$

The same method can be used to obtain $\hat{x}_{p}^{(i)}(k), \hat{P}_{p}(k)$.

\section{Simulation and results}

Drive-by-wire chassis vehicles can realize special working conditions such as U-turn or wedge driving in place, but the driving speed is usually low under these working conditions, which can be verified by common kinematic models. Therefore, through MATLAB/Simulink CarSim simulation, the DUPF algorithm is verified under DLC and weave test. In order to simulate the real signal of the sensor, in the CarSim sensor output port, noise signals are added for the gyroscope longitudinal acceleration, lateral acceleration, yaw rate, wheel output torque, wheel speed, angular acceleration and steer angle. The vehicle parameters are shown in Table 1:

Table 1 Parameters of the tested vehicle

\begin{tabular}{cc}
\hline Parameters (unit) & Value \\
\hline Vehicle mass $(\mathrm{kg})$ & 930 \\
\hline
\end{tabular}




\section{Moment of inertia Izz $\left(\mathrm{kgm}^{2}\right)$}

Center of mass height $(m)$

1460

0.54

Distance from center of mass to

$$
\text { front axle }(m)
$$

Distance from center of mass to rear

$$
\text { axle }(m)
$$

Wheelbase $(m)$

Drag coefficient

\subsection{DLC simulation}

In order to verify the estimation effect of the DUPF algorithm in the longitudinal and lateral directions, a variable-speed DLC is designed. The initial speed is $40 \mathrm{~km} / \mathrm{h}$, output torque of the four wheels is $41 \mathrm{~N} \cdot \mathrm{m}$. The initial value of the vehicle driving state variable is: $x_{s}(0)=[40 / 3.6,0,0,0,0,0]^{T}$, The initial value of the tire force parameter variable is: $x_{p}(0)=$ $[0,0,0,0,0,0,0,0]^{T}$. Road friction coefficient is 0.85 . In order to verify the effectiveness of the algorithm, a vehicle state estimator designed by the commonly used UKF algorithm is introduced for comparison.

(a)

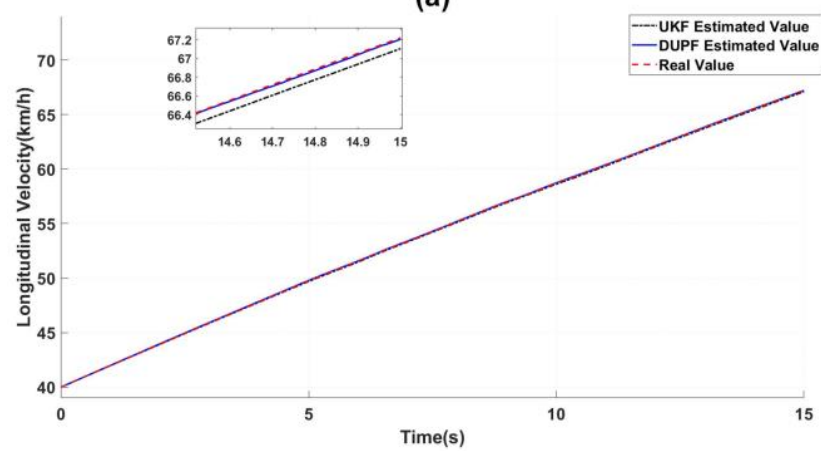

(b)

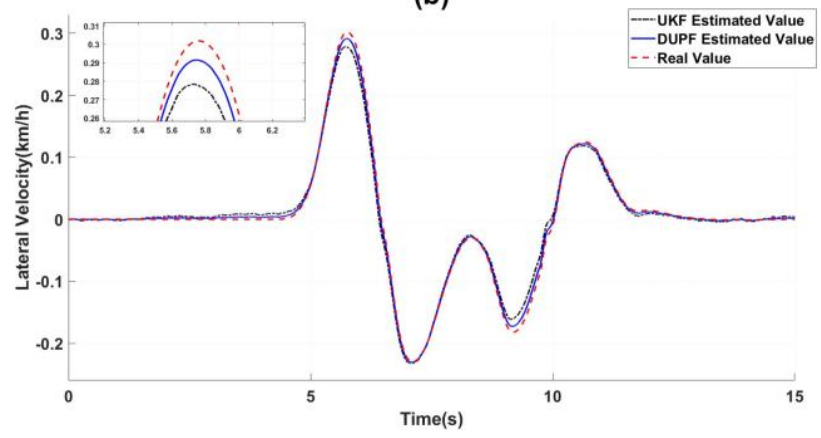

(c)

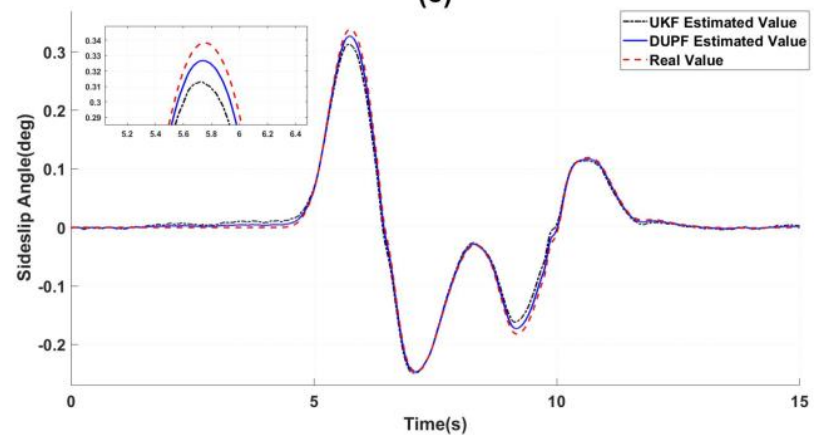

(d)

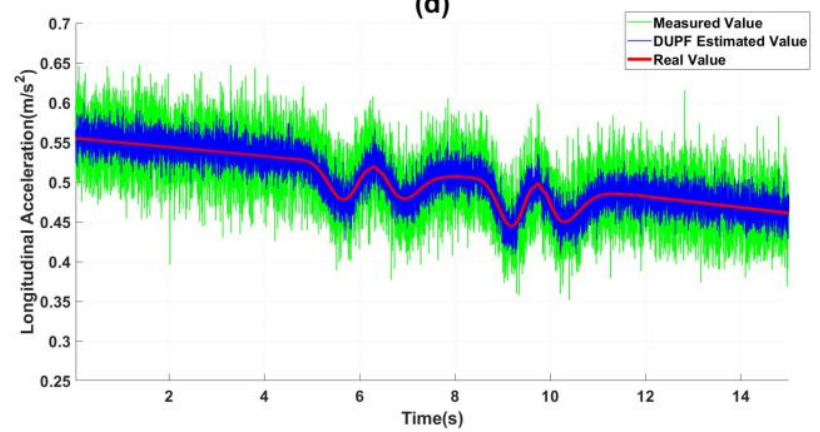

(e)

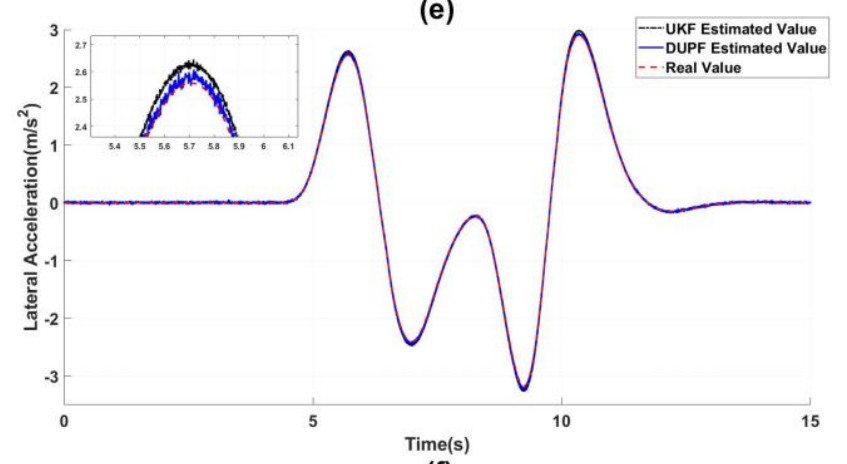

(f)

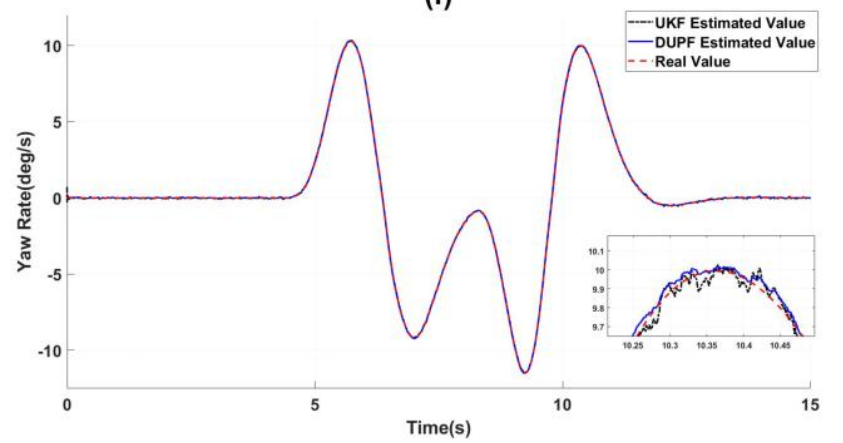

Figure 3 DUPF algorithm estimation result in DLC: (a) longitudinal velocity, (b) lateral velocity, (c) vehicle sideslip angle, (d) longitudinal acceleration, (e) lateral acceleration, (f) yaw rate.

Figure 3 shows the vehicle state observation results obtained by the DUPF and UKF observers. In the longitudinal direction, the two methods are more accurate in estimating the longitudinal vehicle speed, but as the vehicle speed increases, the estimation accuracy of the DUPF algorithm is still high, and the error of the UKF 
algorithm is slightly higher than that of the DUPF algorithm; According to the longitudinal acceleration of the vehicle, it can be found that the DUPF algorithm has a filtering effect on the longitudinal acceleration of the vehicle. In terms of vehicle lateral state estimation, as the vehicle speed increases, the lateral excitation increases, and the errors of the two estimation algorithms increase. The estimation errors of the sideslip angle, lateral velocity, and lateral acceleration gradually increase, but the estimation error of the DUPF algorithm is smaller than UKF algorithm.

In order to illustrate the advantages of the DUPF algorithm more intuitively, the time-average square error (TASE) is used as a reference indicator in the study.[26] TASE can be used to display and compare the estimated performance of the proposed method and the existing method at each Monte Carlo run.

$$
\text { TASE }=\frac{1}{T} \sum_{k=1}^{T}\left(x_{k}^{S}-\hat{x}_{k}^{S}\right)^{2}, \quad s=1, \ldots \ldots, M
$$

Among them, $x_{k}^{S}$ and $\hat{x}_{k}^{S}$ represent the reference vector and the estimated vector in the s-th Monte Carlo, respectively. In this paper, only the TASE results of the DUPF algorithm and the UKF algorithm are compared to further quantify the accuracy of the DUPF algorithm. That is, $x_{k}^{S}$ is the real value of the sensor at time $k$, and $\hat{x}_{k}^{S}$ is the estimated value of the DUPF algorithm and the UKF algorithm at time $k$.

Table 2 Calculated value of vehicle parameter TASE under DLC

\begin{tabular}{ccc}
\hline Calculated parameters & DUPF & UKF \\
\hline Yaw rate & 1.6894 & 2.0084 \\
Longitudinal velocity & 0.6290 & 4.9872 \\
Lateral acceleration & 0.3572 & 1.3962 \\
Lateral velocity & 0.0442 & 0.2015 \\
Sideslip angle & 0.0262 & 0.1347
\end{tabular}

As shown in Table 2, the accuracy of estimation results of the DUPF algorithm are better than the UKF algorithm.

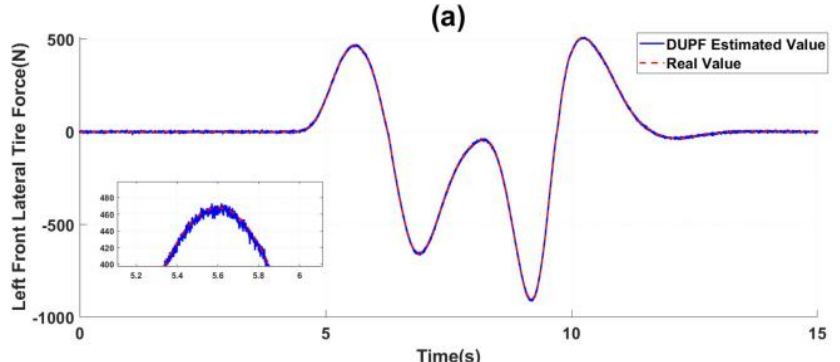

(b)

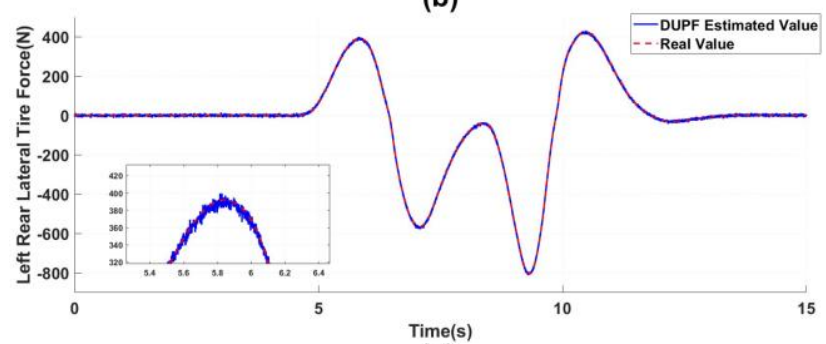

(c)

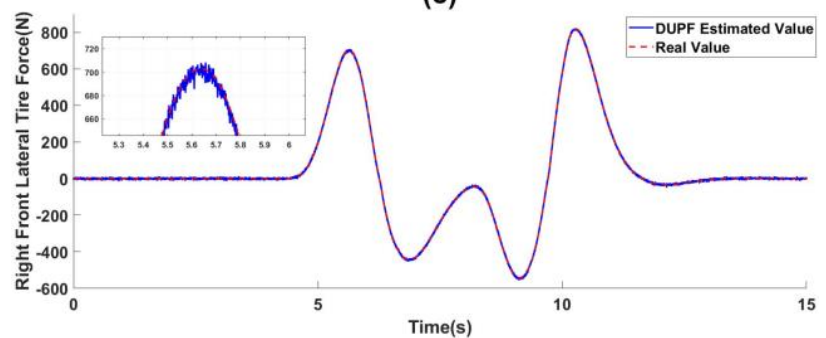

(d)

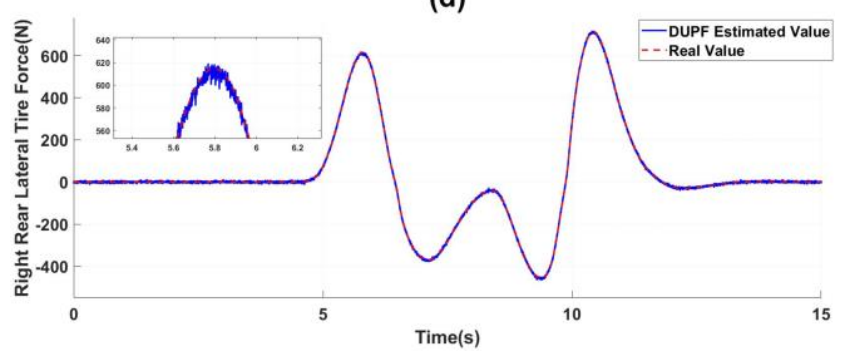

Figure 4 DUPF algorithm estimation result in DLC: (a) left front lateral tire force, (b) left rear lateral tire force, (c) right front lateral tire force, (d) right rear lateral tire force.

As shown in Figure 4, since the drive torque of each wheel of the drive-by-wire chassis vehicle is known, the estimation result obtained by the DUPF algorithm is closer to the true value of the CarSim output, and the error of the estimation result of the tire lateral force is low.

\subsection{Weave test}

To further verify the accuracy of the algorithm's observation in the lateral state, a weave test was set. Among them, the road friction coefficient is set as 0.85 , The wheel steer angle changes with time as a sinusoidal open-loop control, The output torque of the four wheels is set as: $25 \mathrm{~N} \cdot \mathrm{m}$. Prevent the vehicle from side-slip in the process of turning when the speed is too high after accelerating. 
The simulation time is set to $17 \mathrm{~s}$. This working condition also uses the vehicle state observer based on UKF to compare with the DUPF vehicle state observer.

(a)
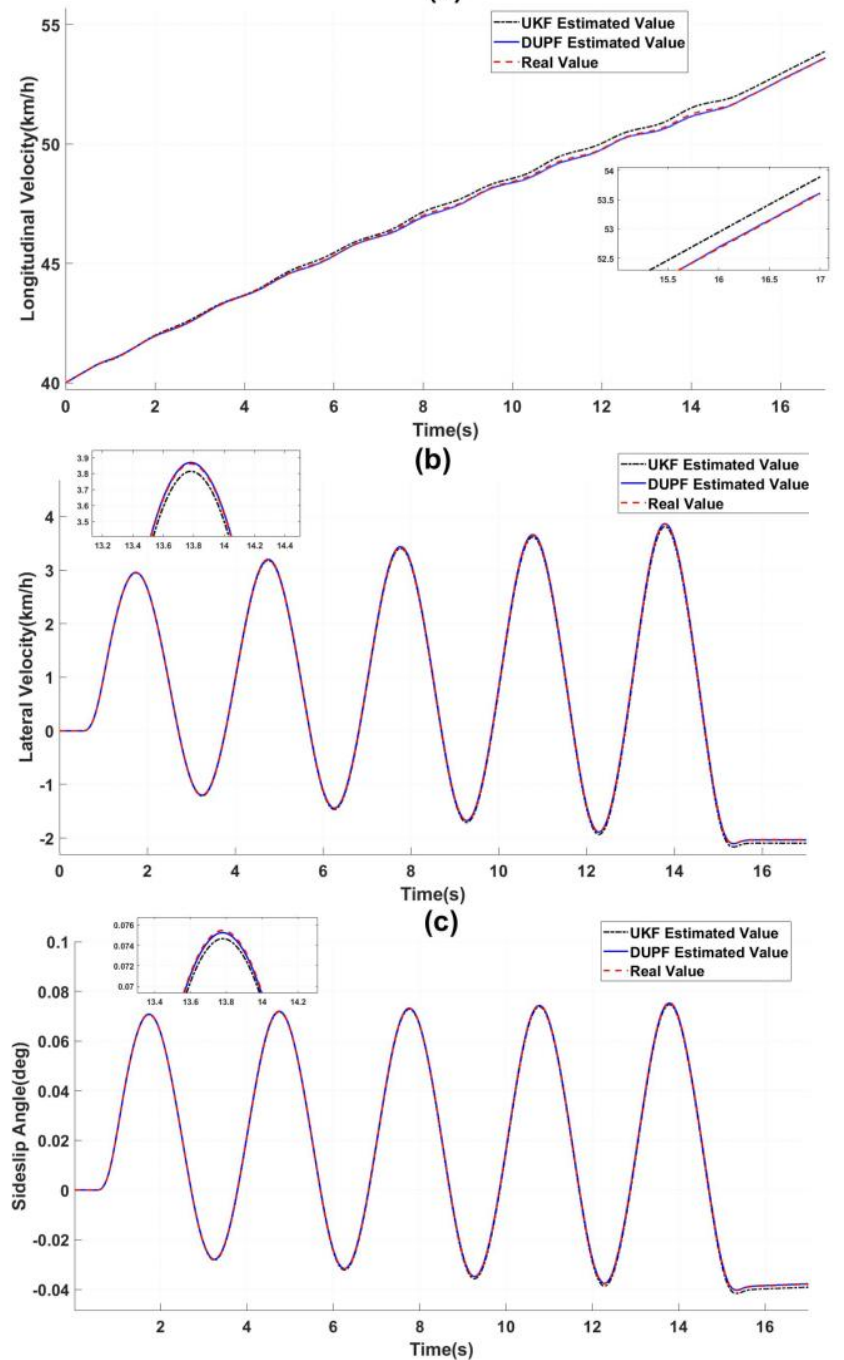

(d)

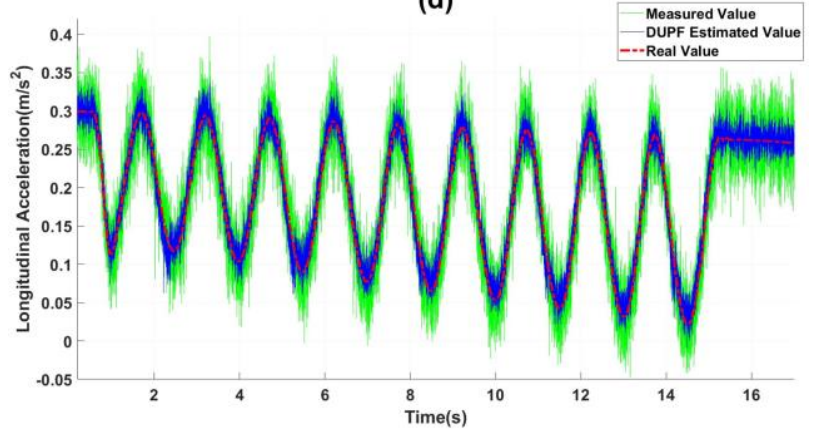

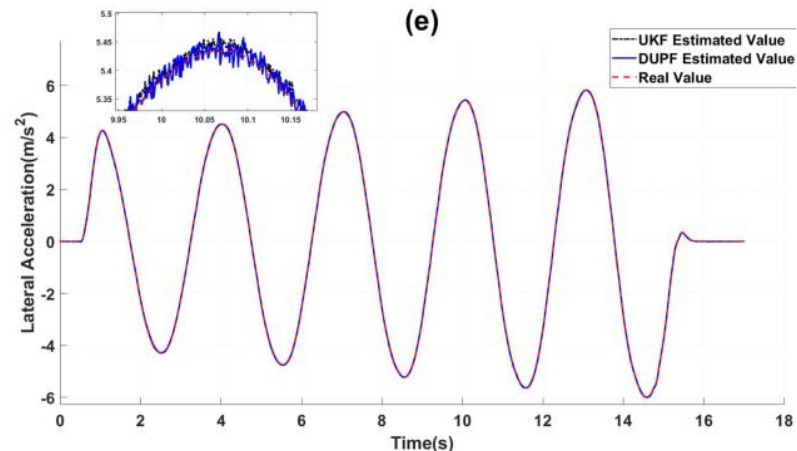

(f)

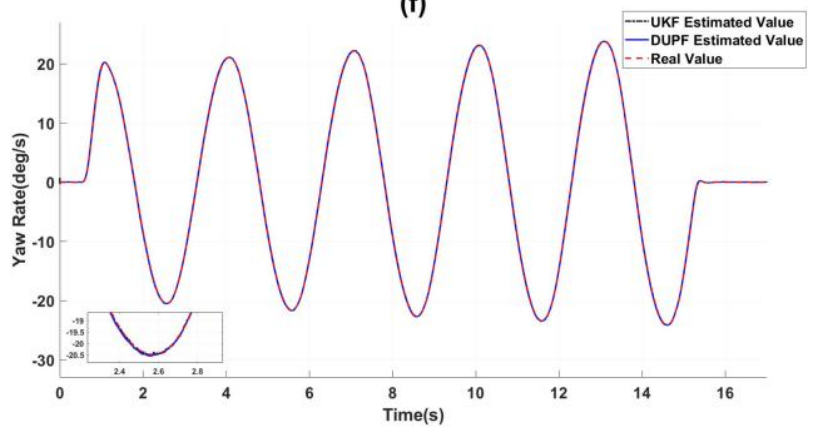

Figure 5 DUPF algorithm estimation result in weave test: (a) longitudinal velocity, (b) lateral velocity, (c) vehicle sideslip angle, (d) longitudinal acceleration, (e) lateral acceleration, (f) yaw rate.

As shown in the Figure 5, it is the estimated result of the vehicle driving state under weave test. In the longitudinal direction, the estimation of longitudinal velocity by the two methods is relatively accurate within $0-5 \mathrm{~s}$. However, as the vehicle longitudinal velocity increases, the estimation accuracy of the DUPF algorithm is still high within 5-17s, and the error of the UKF algorithm is significantly higher than that of the DUPF algorithm. According to the longitudinal acceleration of the vehicle, it can be found that the DUPF algorithm still has a significant filtering effect on the longitudinal acceleration of the vehicle under weave test. In terms of vehicle lateral state estimation, as the vehicle speed increases, the lateral excitation increases, and the errors of the two estimation algorithms increase. There is a certain value error between the lateral velocity and the sideslip angle estimated by the UKF algorithm at $15-17 \mathrm{~s}$ and the actual value of the CarSim output. The main reason is that there is an integral term in the speed estimation process, so there will be a deviation, which can be corrected under appropriate conditions through sensors or GPS and other sensors, and this paper will not introduce it; The vehicle state observer based on the DUPF algorithm and based on the UKF algorithm have low error between the estimated results and the true values such as lateral acceleration and yaw rate which proves that the DUPF algorithm has better performance and robustness. 
(a)

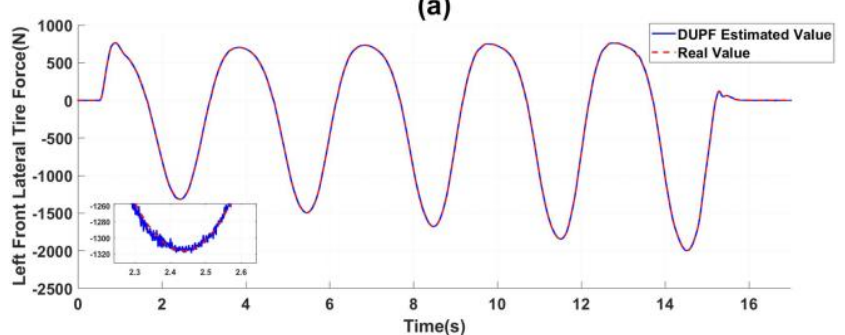

(b)

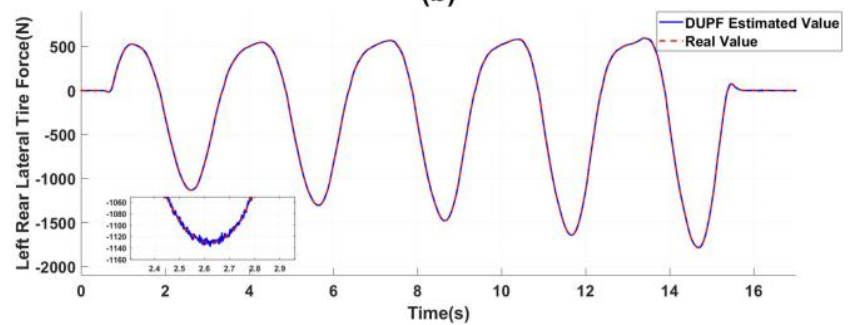

(c)

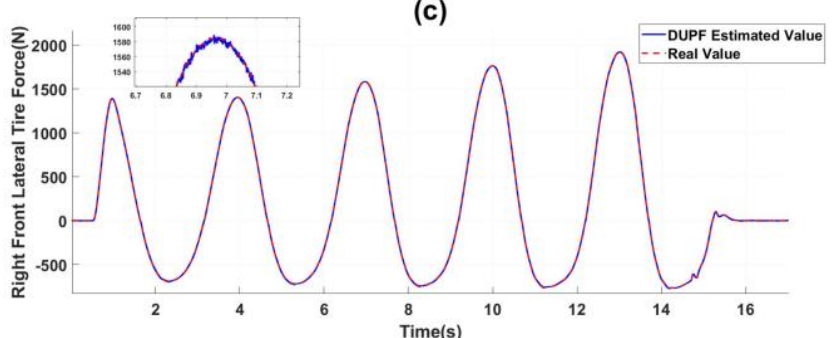

(d)

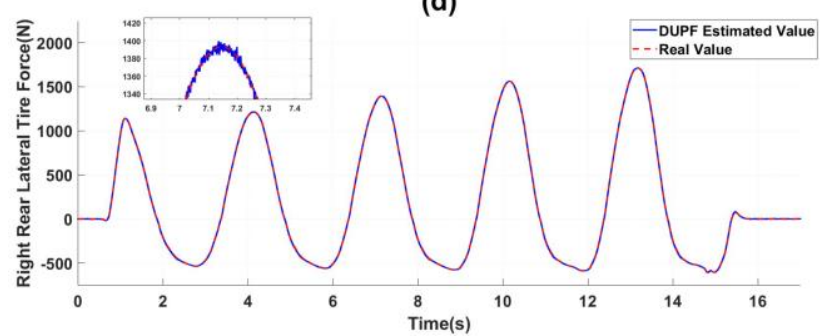

Figure 6 DUPF algorithm estimation result in weave test: (a) left front lateral tire force, (b) left rear lateral tire force, (c) right front lateral tire force, (d) right rear lateral tire force.

As shown in the Figure 6, it is the estimation result of the lateral tire force under the condition of variable speed weave test. It can be seen that the observation result of the lateral force of the vehicle by DUPF is consistent with the actual output value of CarSim.

\subsection{Driving simulator test}

Design a simulator test to evaluate the DUPF algorithm, the simulator test platform is built by CarSimRT, MATLAB/Simulink and dSPACE. After the NI-PXI receives the acceleration, braking, and steering signals controlled by the driver, it processes and analyzes the signals to obtain the torque input and steering angle input of each wheel, and transmits them to the vehicle model in CarSim. Then the sensor signal output in CarSim is processed by the state observer to obtain the vehicle state information, and the real-time video is transmitted back to the display.

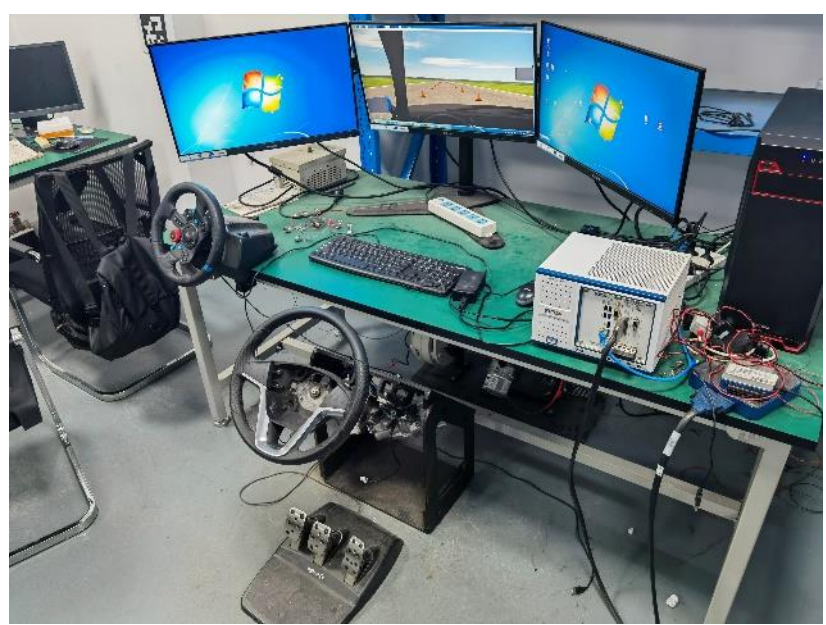

Figure 7 Schematic diagram of online simulation of driving simulator

The driving simulator test adopted a DLC, the coefficient of road adhesion is set as 0.85 , the sampling time period $0-19 \mathrm{~s}$, the sampling time is set to $0.02 \mathrm{~s}$, The number of particles is set to 50 .

(a)

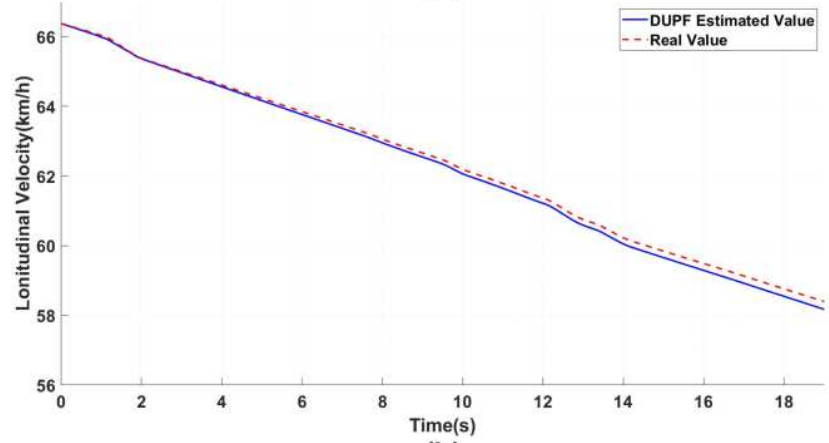

(b)

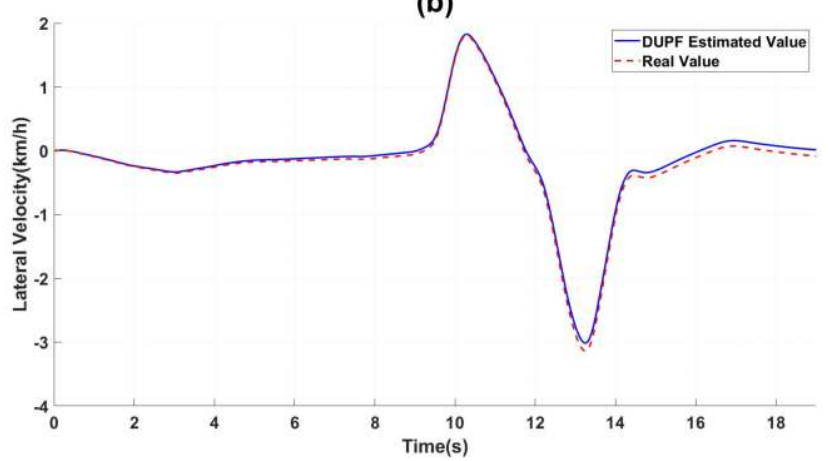


(c)

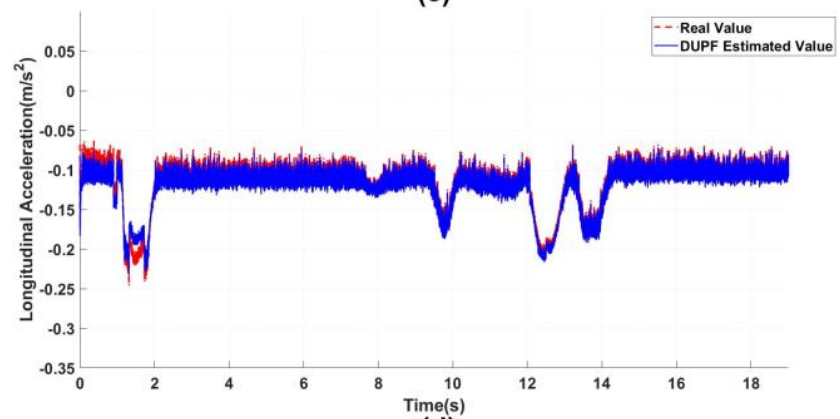

(d)

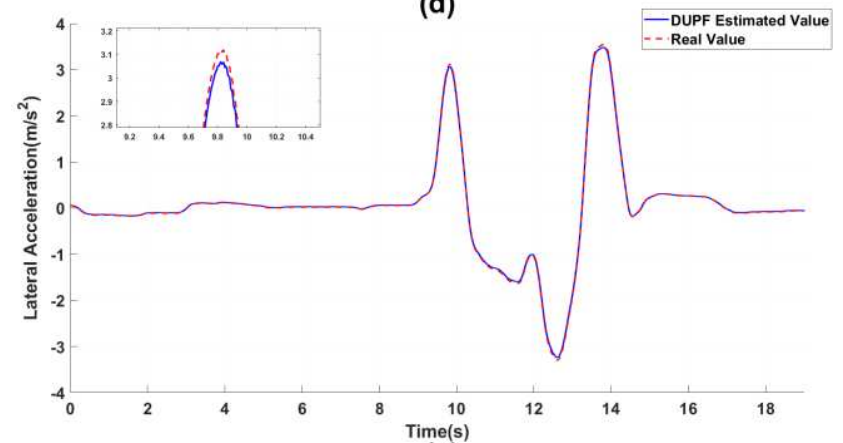

(e)

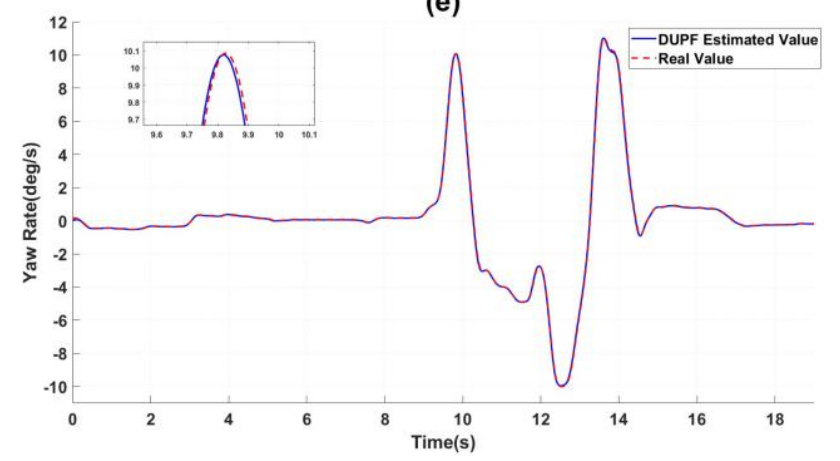

Figure 8 DUPF algorithm estimation result in driving simulator test: (a) longitudinal velocity, (b) lateral velocity, (c) longitudinal acceleration, (d) lateral acceleration, (e) yaw rate.

It can be seen from the Figure 8 that in the longitudinal direction, the estimation result of the longitudinal acceleration is accurate, and the estimation result of the longitudinal velocity has a certain deviation from the true value of the sensor at 12-19s; In the lateral direction, the estimation result of lateral acceleration is also accurate, and the estimation result has some deviations from the true value of the sensor in 14-19s, and the estimation result of the yaw rate is accurate. The main reason for the deviation between the longitudinal velocity and the lateral velocity and the true value is: in the process of solving the velocity, there is a certain integral error, but this part is not within the scope of the algorithm design. (a)

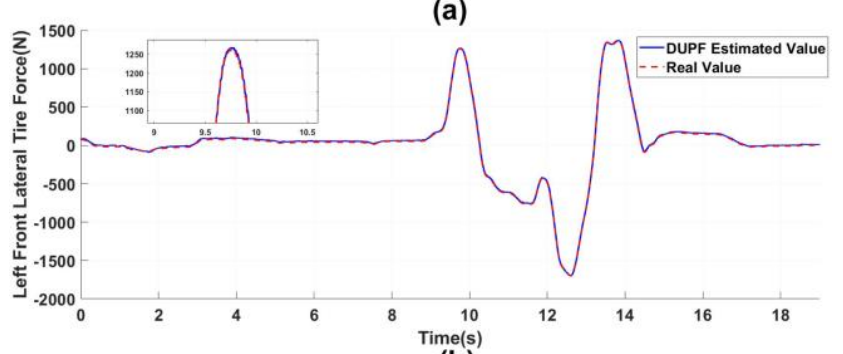

(b)

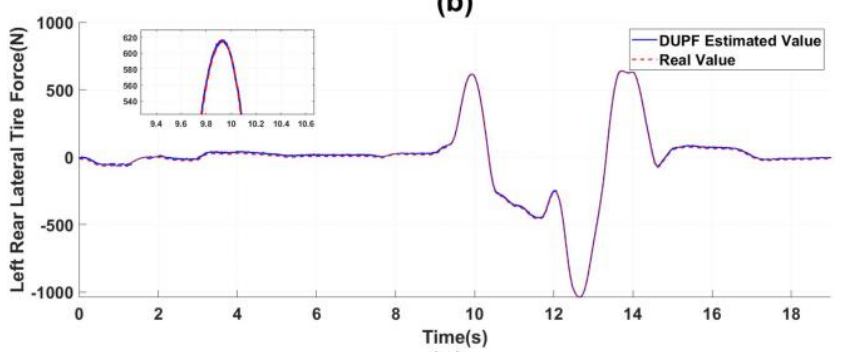

(c)

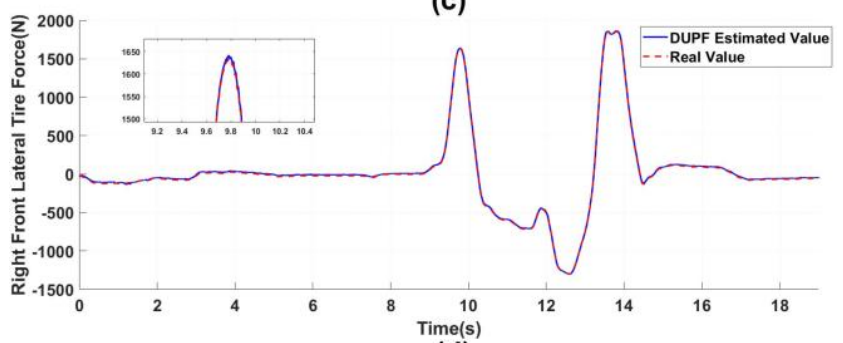

(d)

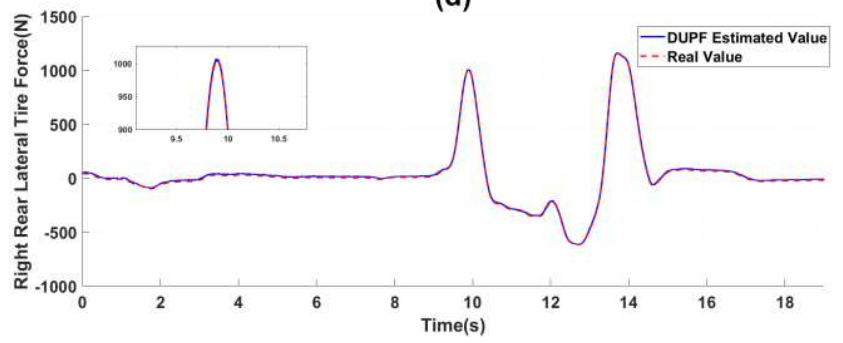

Figure 9 DUPF algorithm estimation result in driving simulator test: (a) left front lateral tire force, (b) left rear lateral tire force, (c) right front lateral tire force, (d) right rear lateral tire force.

It can be obtained from Figure 9, the estimation result of tire lateral force. The estimation result obtained by the DUPF algorithm proposed in this paper has real-time performance.

\section{Conclusion}

Drive-by-wire chassis vehicle with the advantages of precise knowledge of the four-wheel drive torque and independent control of the four-wheel steer angle. This paper proposed a state estimation method for drive-by-wire chassis vehicle based on the DUPF algorithm, and verifies the algorithm through both simulation and experiment.

Inside the DUPF algorithm, UPF estimate the vehicle state information and vehicle parameter information 
respectively. This algorithm structure reduces the uncertainty of parameters and the influence of model parameter changes during driving on the estimation results, and improves the estimation accuracy of vehicle parameters and the robustness of the algorithm. Introduce TASE to quantify the accuracy of the algorithm proposed in this paper. The calculation results show that the accuracy of the DUPF algorithm is higher than that of the UKF algorithm in DLC.

\section{Declaration}

\section{Acknowledgements}

Not applicable.

\section{Funding}

Supported by National Natural Science Foundation of China (Grant No. 51575223) and Jilin Provincial Science and Technology Foundation of China((Grant No. JJKH20200963KJ)

\section{Availability of data and materials}

The datasets supporting the conclusions of this article are included within the article.

\section{Authors' contributions}

The author' contributions are as follows: Z-XW and $\mathrm{H}-\mathrm{YZ}$ were in charge of the whole trial; Z-XW wrote the manuscript; C-NC and QJ assisted with sampling and laboratory analyses. All authors read and approved the final manuscript.

Hong-Yu Zheng, born in 1980, received the B.E. degree in mechanical engineering and automation and the Ph.D.degree in vehicle engineering from Jilin University, Changchun, China, in 2003 and 2009, respectively. From October 2017 to October 2018, he was a Visiting Research Scholar with the Department of Mechanical and Aerospace Engineering, The Ohio State University, Columbus, OH, USA. He is currently a Professor with the State Key Laboratory of Automotive Simulation and Control, Jilin University. His research interests include vehicle dynamics and control and control of autonomous vehicles.

E-mail: zhenghy@jlu.edu.cn

Chuyo Kaku, born in 1962, received the Ph.D.degree in mechanical engineering from Tokyo Institute of Technology, Tokyo, Japan, in 1995 and 1998.

E-mail: guo.kaku@chaoli-electric.com

\section{Competing interests}

The authors declare no competing financial interests.

\section{Consent for publication}

Not applicable

\section{Ethics approval and consent to participate}

Not applicable

\section{References}

[1] J Ni, J B Hu, C L Xiang. A review for design and dynamics control of unmanned ground vehicle. Proceedings of the Institution of Mechanical Engineers, Part D: Journal of Automobile Engineering, 2021, 235(4): 1084-1100.

[2] Y Suzuki, M Takeda. An overview on vehicle lateral dynamics and yaw stability control systems. J. Adv. Vehicle Eng., 2016 2(4): 182-190.

[3] NM Enache, M Netto, S Mammar, et al. Driver steering assistance for lane departure avoidance. Control engineering practice, 2009 , 17(6): 642-651.

[4] M Keller, C Haß, A Seewald, et al. A model predictive approach to emergency maneuvers in critical traffic situations//2015 IEEE 18th International Conference on Intelligent Transportation Systems, Gran Canaria, Spain, Sept 15-18, 2015:369-374.

[5] J Wu, Y H Liu, F B Wang, et al. Vehicle active steering control research based on two-DOF robust internal model control. Chinese Journal of Mechanical Engineering, 2016, 29(4): 739-746.

[6] C F Zong, Z Pan, D Hu, et al. Information fusion algorithm for vehicle state estimation based on extended Kalman filtering. Jixie Gongcheng Xuebao/Journal of Mechanical Engineering, 2009, 45(10): 272-277.

[7] C F Zong, D Hu, X Yang, Z Pan, et al. Vehicle driving state estimation based on extended Kalman filter. Jilin Daxue Xuebao (Gongxueban)/Journal of Jilin University (Engineering and Technology Edition),2009, 39(1): 7-11.

[8] Z G Zhao, H J Chen, J Yang, et al. Estimation of the vehicle speed in the driving mode for a hybrid electric car based on an unscented Kalman filter. Proceedings of the Institution of Mechanical Engineers, Part D: Journal of Automobile Engineering, 2015, 229(4) 437-456.

[9] Q J Cui, R J Ding, B Zhou, et al. Path-tracking of an autonomous vehicle via model predictive control and nonlinear filtering. Proceedings of the Institution of Mechanical Engineers, Part D: Journal of Automobile Engineering,2018, 232(9): 1237-1252.

[10]B Zhang, W Z Zhao, S C Zou, H Zhang, et al. A Reliable Vehicle Lateral Velocity Estimation Methodology based on SBI-LSTM During GPS-outage. IEEE Sensors Journal, 2020, 21(14): 15485-15495.

[11] B Y Zhang, T Xu, H Wang, et al. Vertical Tire Forces Estimation of Multi-Axle Trucks Based on an Adaptive Treble Extend Kalman Filter. Chinese Journal of Mechanical Engineering, 2021, 34(1): $1-19$.

[12] B Lenzo, R D Castro. Vehicle sideslip estimation for four-wheel-steering vehicles using a particle filter//The IAVSD International Symposium on Dynamics of Vehicles on Roads and Tracks, Gothenburg, Sweden, Aug 12-16, 2019: 1624-1634

[13] K Bogdanski, M C Best. Kalman and particle filtering methods for full vehicle and tyre identification. Vehicle System Dynamics, 2018, 56(5): 769-790. 
[14] N Y Ko, T G Kim. Comparison of Kalman filter and particle filter used for localization of an underwater vehicle//2012 9th international conference on ubiquitous robots and ambient intelligence (URAI), Daejeon, Korea (South), Feb 26-28, 2013: 350-352.

[15] G F Tong, Z Fang, X H Xu. A particle swarm optimized particle filter for nonlinear system state estimation//2006 IEEE International Conference on Evolutionary Computation, Vancouver, BC, Sep 16-21, 2006: 438-442.

[16] K Berntorp. Particle filter for combined wheel-slip and vehicle-motion estimation//2015 American Control Conference (ACC), Jul 1-3, 2015: 5414-5419.

[17] D Y Liu, S Huang, S Wu, et al. Direct yaw-moment control of electric vehicle with in-wheel motor drive system. International Journal of Automotive Technology, 2020, 21(4): 1013-1028.

[18] M Lin, J Yoon, B Kim. Self-driving car location estimation based on a particle-aided unscented Kalman filter. Sensors, 2020, 20(9): 2544.

[19] Y H Liu, T Li, Y Y Yang, et al. Estimation of tire-road friction coefficient based on combined APF-IEKF and iteration algorithm. Mechanical Systems and Signal Processing, 2017, 88: 25-35.

[20] Y T Song, H Y Shu, X B Chen. Chassis integrated control for 4WIS distributed drive EVs with model predictive control based on the UKF observer. Science China Technological Sciences, 2020, 63(3): 397-409.

[21] W B Chu, Y G Luo, Y F Dai, et al. In-wheel motor electric vehicle state estimation by using unscented particle filter. International Journal of Vehicle Design, 2015, 67(2): 115-136.

[22] J J Zhu, Z P Wang, L Zhang, et al. State and parameter estimation based on a modified particle filter for an in-wheel-motor-drive electric vehicle. Mechanism and Machine Theory, 2019, 133: 606-624.

[23] X F Pei, Z F Chen, B Yang, et al. Estimation of states and parameters of multi-axle distributed electric vehicle based on dual unscented Kalman filter. Science progress, 2019, 103(1): 1-20.

[24] Z P Wang, J Y Wu, L Zhang, et al. Vehicle sideslip angle estimation for a four-wheel-independent-drive electric vehicle based on a hybrid estimator and a moving polynomial Kalman smoother. Proceedings of the Institution of Mechanical Engineers, Part K: Journal of Multi-body Dynamics, 2018, 233(1): 125-140.

[25] H Pacejka. Tire and vehicle dynamics. Elsevier. 2005.

[26] Y L Huang, Y G Zhang, N Li, et al. Design of Gaussian approximate filter and smoother for nonlinear systems with correlated noises at one epoch apart. Circuits, Systems, and Signal Processing, 2016, 35(11): 3981-4008.

\section{Biographical notes}

Zi-Xu Wang, born in 1999, is currently a master candidate at State Key Laboratory of Automotive Simulation and Control, Jilin University, Changchun, China

Tel: +86-18343526155; E-mail: 345197599@qq.com

Chao-Ning Chen, born in 1999, is currently a Ph.D candidate at State Key Laboratory of Automotive Simulation and Control, Jilin University, Changchun, China.

E-mail:1176660329@qq.com

Quan Jiang, born in 1997, is currently a master candidate at State Key Laboratory of Automotive Simulation and Control, Jilin University, Changchun, China
E-mail:1049819809@qq.com@163.com

Hong-Yu Zheng, born in 1980, received the B.E. degree in mechanical engineering and automation and the $\mathrm{Ph} . \mathrm{D}$.degree in vehicle engineering from Jilin University, Changchun, China, in 2003 and 2009, respectively. From October 2017 to October 2018, he was a Visiting Research Scholar with the Department of Mechanical and Aerospace Engineering, The Ohio State University, Columbus, OH, USA. He is currently a Professor with the State Key Laboratory of Automotive Simulation and Control, Jilin University. His research interests include vehicle dynamics and control and control of autonomous vehicles.

E-mail: zhenghy@jlu.edu.cn

Chuyo Kaku, born in 1962, received the Ph.D.degree in mechanical engineering from Tokyo Institute of Technology, Tokyo, Japan, in 1995 and 1998.

E-mail: guo.kaku@chaoli-electric.com 